\title{
Self-inductance coefficient for toroidal thin conductors
}

\author{
Y. Amirat and R. Touzani \\ Laboratoire de Mathématiques Appliquées, UMR CNRS 6620 \\ Université Blaise Pascal (Clermont-Ferrand 2) \\ 63177 Aubière cedex, France
}

\begin{abstract}
We consider the inductance coefficient for a thin toroidal inductor whose thickness depends on a small parameter $\varepsilon>0$. An explicit form of the singular part of the corresponding potential $u^{\varepsilon}$ is given. This allows to construct the limit potential $u$ (as $\varepsilon \rightarrow 0$ ) and an approximation of the inductance coefficient $L^{\varepsilon}$. We establish some estimates of the deviation $u^{\varepsilon}-u$ and of the error of approximation of the inductance. The main result shows that $L^{\varepsilon}$ behaves asymptotically as $\ln \varepsilon$, when $\varepsilon \rightarrow 0$.
\end{abstract}

Key words: Asymptotic behaviour, self inductance, eddy currents, thin domain

\section{Introduction}

In electrotechnical engineering, eddy current devices often involve thick conductors in which a magnetic field is induced, and circuits made of thin wires or coils, as inductors, connected to a power source generator. Mathematical modelling of such devices has then to take into account the simultaneous presence of thick conductors and thin inductors. For a two-dimensional configuration where the magnetic field has only one nonvanishing component, it was shown that the eddy current equation has the Kirchhoff circuit equation as a limit problem, as the thickness of the inductor tends to zero, see [1]. For the threedimensional case, eddy current models require the use of a relevant quantity that is the self-inductance of the inductor, see [2], [3]. This number has to be evaluated a priori as a part of problem data. It is the purpose of the present

Email addresses: Youcef . Amirat@math.univ-bpclermont.fr (Y. Amirat), Rachid.Touzani@math.univ-bpclermont.fr (R. Touzani). 
paper to study the asymptotic behaviour of this number when the thickness of the inductor goes to zero.

Let us consider a toroidal domain of $\mathbb{R}^{3}$, denoted by $\Omega_{\varepsilon}$, whose thickness depends on a small parameter $\varepsilon>0$. The geometry of $\Omega_{\varepsilon}$ will be described in the next section. We denote by $\Gamma_{\varepsilon}$ the boundary of $\Omega_{\varepsilon}$, by $n_{\varepsilon}$ the outward unit normal to $\Gamma_{\varepsilon}$, and by $\Omega_{\varepsilon}^{\prime}$ the complement of its closure, that is $\Omega_{\varepsilon}^{\prime}=\mathbb{R}^{3} \backslash \bar{\Omega}_{\varepsilon}$. We denote by $\Sigma$ a cut in the domain $\Omega_{\varepsilon}^{\prime}$, that is, $\Sigma$ is a smooth orientable surface such that, for any $\varepsilon>0, \Omega_{\varepsilon}^{\prime} \backslash \Sigma$ is simply connected.

Let now $\boldsymbol{h}^{\varepsilon}$ denote the time-harmonic and complex-valued magnetic field. Neglecting the displacement currents, it follows from Maxwell's equations that

$$
\operatorname{curl} \boldsymbol{h}^{\varepsilon}=0, \operatorname{div} \boldsymbol{h}^{\varepsilon}=0 \quad \text { in } \Omega_{\varepsilon}^{\prime} .
$$

Then, by a result in [4], p. 265, $\boldsymbol{h}^{\varepsilon}$ may be written in the form

$$
\boldsymbol{h}_{\mid \Omega_{\varepsilon}^{\prime}}^{\varepsilon}=\nabla \varphi^{\varepsilon}+I^{\varepsilon} \nabla u^{\varepsilon}
$$

where $I^{\varepsilon}$ is a complex number, $\varphi^{\varepsilon} \in W^{1}\left(\Omega_{\varepsilon}^{\prime}\right)$ and satisfies

$$
\Delta \varphi^{\varepsilon}=0 \quad \text { in } \Omega_{\varepsilon}^{\prime},
$$

and $u^{\varepsilon}$ is solution of :

$$
\begin{cases}\Delta u^{\varepsilon}=0 & \text { in } \Omega_{\varepsilon}^{\prime} \backslash \Sigma, \\ \frac{\partial u^{\varepsilon}}{\partial n}=0 & \text { on } \Gamma_{\varepsilon}, \\ {\left[u^{\varepsilon}\right]_{\Sigma}=1,} & \\ {\left[\frac{\partial u^{\varepsilon}}{\partial n}\right]_{\Sigma}=0 .} & \end{cases}
$$

Here $W^{1}\left(\Omega_{\varepsilon}^{\prime}\right)$ is the Sobolev space

$$
W^{1}\left(\Omega_{\varepsilon}^{\prime}\right)=\left\{v ; \rho v \in L^{2}\left(\Omega_{\varepsilon}^{\prime}\right), \nabla v \in \boldsymbol{L}^{2}\left(\Omega_{\varepsilon}^{\prime}\right)\right\},
$$

equipped with the norm

$$
\|v\|_{W^{1}\left(\Omega_{\varepsilon}^{\prime}\right)}=\left(\|\rho v\|_{L^{2}\left(\Omega_{\varepsilon}^{\prime}\right)}^{2}+\|\nabla v\|_{L^{2}\left(\Omega_{\varepsilon}^{\prime}\right)}^{2}\right)^{\frac{1}{2}}
$$

where $\boldsymbol{L}^{p}\left(\Omega_{\varepsilon}^{\prime}\right)$ denotes the space $L^{p}\left(\Omega_{\varepsilon}^{\prime}\right)^{3}$ and $\rho$ is the weight function $\rho(\boldsymbol{x})=$ $\left(1+|\boldsymbol{x}|^{2}\right)^{-\frac{1}{2}}$. Let us note here, see [4], pp. 649-651, that

$$
|v|_{W^{1}\left(\Omega_{\varepsilon}^{\prime}\right)}=\left(\int_{\Omega_{\varepsilon}^{\prime}}|\nabla v|^{2} d \boldsymbol{x}\right)^{\frac{1}{2}}
$$


is a norm on $W^{1}\left(\Omega_{\varepsilon}^{\prime}\right)$, equivalent to (3). In (2), $n$ is the unit normal on $\Sigma$, and $\left[u^{\varepsilon}\right]_{\Sigma}$ (resp. $\left.\left[\frac{\partial u^{\varepsilon}}{\partial n}\right]_{\Sigma}\right)$ denotes the jump of $u^{\varepsilon}$ (resp. $\frac{\partial u^{\varepsilon}}{\partial n}$ ) across $\Sigma$.

In (1), the number $I^{\varepsilon}$ can be interpreted as the total current flowing in the inductor, see [3].

The inductance coefficient is then defined by the expression

$$
L^{\varepsilon}=\int_{\Omega_{\varepsilon}^{\prime} \backslash \Sigma}\left|\nabla u^{\varepsilon}\right|^{2} d \boldsymbol{x} .
$$

Our goal is to study the asymptotic behaviour of $u^{\varepsilon}$ and $L^{\varepsilon}$ as $\varepsilon$ goes to zero. We first give an explicit form of the singular part of the potential $u^{\varepsilon}$ which allows to construct the limit potential $u$ (as $\varepsilon \rightarrow 0$ ) and an approximation of the inductance $L^{\varepsilon}$. We then prove that the deviation $\left\|u^{\varepsilon}-u\right\|_{W^{1}\left(\Omega_{\varepsilon}^{\prime}\right)}$ and the error of approximation of $L^{\varepsilon}$ are of order $O\left(\varepsilon^{\frac{5}{6}-\eta}\right)$ for every $\eta>0$. Finally we show that the inductance coefficient $L^{\varepsilon}$ behaves asymptotically as $\ln \varepsilon$, when $\varepsilon \rightarrow 0$, and we thus recover the result stated (without proof) in [5], p. 137 .

Let us outline the organization of this paper. In Section 2 we specify the geometry of the inductor, assuming that it is a toroidal neighborhood of a closed curve, the internal radius of the torus being proportional to a small positive number $\varepsilon$. Section 3 states the main result and gives the main steps in its proof. Let us note here that an extended version of this paper with detailed proofs can be consulted in [6].

\section{Geometry of the domain}

We consider a toroidal domain, with a small cross section. This domain may be defined as a tubular neighborhood of a closed curve. Let $\gamma$ denote a closed Jordan arc of class $\mathcal{C}^{3}$ in $\mathbb{R}^{3}$, with a parametric representation defined by a function $\boldsymbol{g}:[0,1] \rightarrow \mathbb{R}^{3}$ satisfying

$$
\boldsymbol{g}(0)=\boldsymbol{g}(1), \boldsymbol{g}^{\prime}(0)=\boldsymbol{g}^{\prime}(1),\left|\boldsymbol{g}^{\prime}(s)\right| \geq C_{0}>0 .
$$

For each $s \in(0,1]$ we denote by $(\boldsymbol{t}(s), \boldsymbol{\nu}(s), \boldsymbol{b}(s))$ the Serret-Frénet coordinates at the point $\boldsymbol{g}(s)$, i.e., $\boldsymbol{t}(s), \boldsymbol{\nu}(s), \boldsymbol{b}(s)$ are respectively the unit tangent vector to $\gamma$, the principal normal and the binormal, given by

$$
\boldsymbol{t}=\frac{\boldsymbol{g}^{\prime}}{\left|\boldsymbol{g}^{\prime}\right|}, \boldsymbol{\nu}=\frac{\boldsymbol{t}^{\prime}}{\left|\boldsymbol{t}^{\prime}\right|}, \boldsymbol{b}=\boldsymbol{t} \times \boldsymbol{\nu}
$$

and by $\kappa$ and $\tau$ respectively the curvature and the torsion of the $\operatorname{arc} \gamma$. 
Let $\widehat{\Omega}=(0,1)^{2} \times(0,2 \pi)$ and let $\delta$ denote a positive number to be chosen in a convenient way. We define, for any $\varepsilon, 0 \leq \varepsilon<\delta$, the mapping $\boldsymbol{F}_{\varepsilon}: \widehat{\Omega} \rightarrow \mathbb{R}^{3}$ by

$$
\boldsymbol{F}_{\varepsilon}(s, \xi, \theta)=\boldsymbol{g}(s)+r_{\varepsilon}(\xi)(\cos \theta \boldsymbol{\nu}(s)+\sin \theta \boldsymbol{b}(s)),
$$

where $r_{\varepsilon}(\xi)=(\delta-\varepsilon) \xi+\varepsilon$. The jacobian of $\boldsymbol{F}_{\varepsilon}$ is therefore given by

$$
J_{\varepsilon}(s, \xi, \theta)=(\delta-\varepsilon) a_{\varepsilon}(s, \xi, \theta) r_{\varepsilon}(\xi),
$$

where

$$
a_{\varepsilon}(s, \xi, \theta)=\left|\boldsymbol{g}^{\prime}(s)\right|-r_{\varepsilon}(\xi) \kappa(s) \cos \theta \text {. }
$$

According to (4), if $\delta$ is chosen such that

$$
\delta|\kappa(s)|<\left|\boldsymbol{g}^{\prime}(s)\right|, \quad 0 \leq s \leq 1
$$

then

$$
0<C_{1} \leq a_{\varepsilon} \leq C_{2},
$$

and the mapping $\boldsymbol{F}_{\varepsilon}$ is a $\mathcal{C}^{1}$-diffeomorphism from $\widehat{\Omega}$ into $\Lambda_{\varepsilon}^{\delta}=\boldsymbol{F}_{\varepsilon}(\widehat{\Omega})$.

Here and in the sequel, the quantities $C, C_{1}, C_{2}, \ldots$ denote generic positive numbers that do not depend on $\varepsilon$.

We now set, for any $0<\varepsilon<\delta$,

$$
\Omega_{\delta}=\Lambda_{0}^{\delta}=\boldsymbol{F}_{0}(\widehat{\Omega}), \Omega_{\delta}^{\prime}=\mathbb{R}^{3} \backslash \bar{\Omega}_{\delta}, \Omega_{\varepsilon}^{\prime}=\operatorname{Int}\left(\bar{\Omega}_{\delta}^{\prime} \cup \bar{\Lambda}_{\varepsilon}^{\delta}\right), \Omega_{\varepsilon}=\mathbb{R}^{3} \backslash \bar{\Omega}_{\varepsilon}^{\prime} .
$$

For technical reasons, we choose in the sequel $0<\varepsilon \leq \frac{\delta}{2}$.

Given a function $v$ on $\Lambda_{\varepsilon}^{\delta}$, we define the function $\widehat{v}$ on $\widehat{\Omega}$ by $\widehat{v}=v \circ \boldsymbol{F}_{\varepsilon}$. If $v \in L^{p}\left(\Lambda_{\varepsilon}^{\delta}\right), 1 \leq p \leq \infty$, then $\widehat{v} \in L^{p}(\widehat{\Omega})$ and we have

$$
\int_{\Lambda_{\varepsilon}^{\delta}} v d \boldsymbol{x}=\int_{\widehat{\Omega}} \widehat{v}(\delta-\varepsilon) a_{\varepsilon} r_{\varepsilon} d \widehat{\boldsymbol{x}} .
$$

Moreover, for $u$ and $v$ in $H^{1}\left(\Lambda_{\varepsilon}^{\delta}\right)$, we have

$$
\begin{aligned}
\int_{\Lambda_{\varepsilon}^{\delta}} \nabla u \cdot \nabla v d \boldsymbol{x}=(\delta-\varepsilon) \int_{\widehat{\Omega}} & \left(\frac{r_{\varepsilon}}{a_{\varepsilon}} \frac{\partial \widehat{u}}{\partial s} \frac{\partial \widehat{v}}{\partial s}+\frac{r_{\varepsilon} a_{\varepsilon}}{(\delta-\varepsilon)^{2}} \frac{\partial \widehat{u}}{\partial \xi} \frac{\partial \widehat{v}}{\partial \xi}\right. \\
& +\left(\frac{a_{\varepsilon}}{r_{\varepsilon}}+\frac{\tau^{2} r_{\varepsilon}}{a_{\varepsilon}}\right) \frac{\partial \widehat{u}}{\partial \theta} \frac{\partial \widehat{v}}{\partial \theta} \\
& \left.-\frac{r_{\varepsilon} \tau}{a_{\varepsilon}}\left(\frac{\partial \widehat{u}}{\partial s} \frac{\partial \widehat{v}}{\partial \theta}+\frac{\partial \widehat{u}}{\partial \theta} \frac{\partial \widehat{v}}{\partial s}\right)\right) d \widehat{\boldsymbol{x}} .
\end{aligned}
$$

We also define the set $\widehat{\Gamma}=(0,1) \times(0,2 \pi)$ and the mapping $\boldsymbol{G}_{\varepsilon}: \widehat{\Gamma} \rightarrow \mathbb{R}^{3}$ by

$$
\boldsymbol{G}_{\varepsilon}(s, \theta)=\boldsymbol{g}(s)+\varepsilon(\cos \theta \boldsymbol{\nu}(s)+\sin \theta \boldsymbol{b}(s)) \text {. }
$$


The boundary of $\Omega_{\varepsilon}^{\prime}$ is then represented by $\Gamma_{\varepsilon}=\overline{\boldsymbol{G}_{\varepsilon}(\widehat{\Gamma})}$. If $w \in L^{2}\left(\Gamma_{\varepsilon}\right)$, we define $\widehat{w} \in L^{2}(\widehat{\Gamma})$ by $\widehat{w}=w \circ \boldsymbol{G}_{\varepsilon}$, and we have

$$
\int_{\Gamma_{\varepsilon}} w d \sigma=\int_{\widehat{\Gamma}} \widehat{w} \varepsilon\left(\left|\boldsymbol{g}^{\prime}\right|-\varepsilon \kappa \cos \theta\right) d \widehat{\sigma} .
$$

Clearly, $\Omega_{\varepsilon}$ and its complement $\Omega_{\varepsilon}^{\prime}$ are connected domains but they are not simply connected. To define a cut in $\Omega_{\varepsilon}^{\prime}$, we denote by $\Sigma_{0}$ the set $\boldsymbol{F}_{0}\left((0,1)^{2} \times\right.$ $\{0\})$ and $\partial \Sigma_{0}=\boldsymbol{F}_{0}((0,1) \times\{1\} \times\{0\})$. Let $\Sigma^{\prime}$ denote a smooth simple surface that has $\partial \Sigma_{0}$ as a boundary and such that the surface $\Sigma=\Sigma^{\prime} \cup \Sigma_{0}$ is oriented and of class $\mathcal{C}^{1}$ (cf. [7]). We denote by $\Sigma^{+}$(resp. $\Sigma^{-}$) the oriented surface with positive (resp. negative) orientation, and by $n$ the unit normal on $\Sigma$ directed from $\Sigma^{+}$to $\Sigma^{-}$. If $w \in W^{1}\left(\mathbb{R}^{3} \backslash \Sigma\right)$, we denote by $[w]_{\Sigma}$ the jump of $w$ across $\Sigma$ in the direction of $n$, i.e.

$$
[w]_{\Sigma}=w_{\mid \Sigma^{+}}-w_{\mid \Sigma^{-}} .
$$

\section{Formulation of the problem and statement of the result}

We consider the boundary value problem

$$
\begin{cases}\Delta u^{\varepsilon}=0 & \text { in } \Omega_{\varepsilon}^{\prime} \backslash \Sigma, \\ \frac{\partial u^{\varepsilon}}{\partial n_{\varepsilon}}=0 & \text { on } \Gamma_{\varepsilon}, \\ {\left[u^{\varepsilon}\right]_{\Sigma}=1,} & \\ {\left[\frac{\partial u^{\varepsilon}}{\partial n}\right]_{\Sigma}=0,} & \end{cases}
$$

where $n_{\varepsilon}$ denotes the unit normal on $\Gamma_{\varepsilon}$ pointing outward $\Omega_{\varepsilon}^{\prime}$ and $\boldsymbol{n}$ is the unit normal on $\Sigma$ oriented from $\Sigma^{+}$toward $\Sigma^{-}$. The inductance coefficient is defined by

$$
L^{\varepsilon}=\int_{\Omega_{\varepsilon}^{\prime} \backslash \Sigma}\left|\nabla u^{\varepsilon}\right|^{2} d \boldsymbol{x} .
$$

We want to describe the asymptotic behaviour of $u^{\varepsilon}$ and $L^{\varepsilon}$ as $\varepsilon \rightarrow 0$.

We first exhibit a function that has the same singularity as might be expected for the solution of Problem (5) (as $\varepsilon \rightarrow 0$ ). Let us define

$$
\widehat{v}(s, \xi, \theta)=\frac{\theta}{2 \pi} \widehat{\varphi}(\xi), \quad(s, \xi, \theta) \in \widehat{\Omega}
$$

where $\hat{\varphi} \in \mathcal{C}^{2}(\mathbb{R})$ and such that

$$
\widehat{\varphi}(\xi)=1 \text { for } 0 \leq \xi \leq \frac{1}{2}, \quad \widehat{\varphi}(\xi)=0 \text { for } \xi \geq \frac{3}{4} .
$$


We then define $v: \mathbb{R}^{3} \rightarrow \mathbb{R}$ by :

$$
v(\boldsymbol{x})= \begin{cases}\widehat{v}\left(\boldsymbol{F}_{0}^{-1}(\boldsymbol{x})\right) & \text { if } \boldsymbol{x} \in \Omega_{\delta} \\ 0 & \text { if } \boldsymbol{x} \in \Omega_{\delta}^{\prime}\end{cases}
$$

Let us also define

$$
\begin{aligned}
& \widehat{f}(s, \xi, \theta)=\frac{1}{2 \pi a_{0}}\left(\frac{\kappa \sin \theta}{\delta \xi}-\frac{\tau^{2} \delta \xi \kappa \sin \theta}{a_{0}^{2}}-\frac{\partial}{\partial s}\left(\frac{\tau}{a_{0}}\right)\right) \widehat{\varphi} \\
& f(\boldsymbol{x})= \begin{cases}\widehat{f}\left(\boldsymbol{F}_{0}^{-1}(\boldsymbol{x})\right) & \text { if } \boldsymbol{x} \in \Omega_{\delta}, \\
0 & \text { if } \boldsymbol{x} \in \Omega_{\delta}^{\prime},\end{cases} \\
& \varphi(\boldsymbol{x})= \begin{cases}\widehat{\varphi}(\xi) & \text { if } \boldsymbol{x} \in \Omega_{\delta}, \\
0 & \text { if } \boldsymbol{x} \in \Omega_{\delta}^{\prime} .\end{cases}
\end{aligned}
$$

By straightforward calculations, we see that function $v$ is solution of

$$
\left\{\begin{array}{l}
\Delta v=f \quad \text { in } \mathbb{R}^{3} \backslash \Sigma \\
{[v]_{\Sigma}=\varphi,} \\
{\left[\frac{\partial v}{\partial n}\right]_{\Sigma}=0 .}
\end{array}\right.
$$

Moreover, it satisfies

$$
\frac{\partial v}{\partial n_{\varepsilon}}=0 \quad \text { on } \Gamma_{\varepsilon} .
$$

Furthermore, we have for any $1 \leq p<2$,

$$
f \in L^{p}\left(\mathbb{R}^{3}\right), v \in L^{\infty}\left(\mathbb{R}^{3}\right) \cap W^{1, p}\left(\mathbb{R}^{3} \backslash \Sigma\right) .
$$

We note here that $v \notin H^{1}\left(\mathbb{R}^{3} \backslash \Sigma\right)$. However, for any $\varepsilon, v \in H^{1}\left(\Omega_{\varepsilon}^{\prime} \backslash \Sigma\right)$.

Let us now set $w^{\varepsilon}=u^{\varepsilon}-v$. We have by subtracting (7) from (5),

$$
\begin{cases}-\Delta w^{\varepsilon}=f & \text { in } \Omega_{\varepsilon}^{\prime} \backslash \Sigma, \\ \frac{\partial w^{\varepsilon}}{\partial n_{\varepsilon}}=0 & \text { on } \Gamma_{\varepsilon} \\ {\left[w^{\varepsilon}\right]_{\Sigma}=1-\varphi,} & \\ {\left[\frac{\partial w^{\varepsilon}}{\partial n}\right]_{\Sigma}=0 .} & \end{cases}
$$

We note here that Problem (8) differs from (5) by the value of the jump of the solution across $\Sigma$ and by the presence of a right-hand side $f$. However, we notice that $1-\varphi$ vanishes in a neighborhood of $\partial \Sigma$ and then, for Problem (8), the jump of $w^{\varepsilon}$ vanishes in a neighborhood of $\partial \Sigma$. 
Now, to study the asymptotic behaviour of $w^{\varepsilon}$ and $L^{\varepsilon}$ as $\varepsilon \rightarrow 0$ we consider the following decomposition. Let $w_{1}$ denote the solution of

$$
\begin{cases}\Delta w_{1}=0 & \text { in } \mathbb{R}^{3} \backslash \Sigma, \\ {\left[w_{1}\right]_{\Sigma}=1-\varphi,} & \\ {\left[\frac{\partial w_{1}}{\partial n}\right]_{\Sigma}=0,} & \\ w_{1}(\boldsymbol{x})=O\left(|\boldsymbol{x}|^{-1}\right) & |\boldsymbol{x}| \rightarrow \infty .\end{cases}
$$

Using [4], p. 654, and the fact that $1-\varphi$ vanishes in a neighborhood of $\partial \Sigma$, we see that Problem (9) has a unique solution in $W^{1}\left(\mathbb{R}^{3} \backslash \Sigma\right)$ given by

$$
w_{1}(\boldsymbol{x})=\frac{1}{4 \pi} \int_{\Sigma}(1-\varphi(\boldsymbol{y})) \frac{\boldsymbol{n}(\boldsymbol{y}) \cdot(\boldsymbol{x}-\boldsymbol{y})}{|\boldsymbol{x}-\boldsymbol{y}|^{3}} d \sigma(\boldsymbol{y}), \quad \boldsymbol{x} \in \mathbb{R}^{3} \backslash \Sigma .
$$

Then we write $w^{\varepsilon}=w_{1}+w_{2}^{\varepsilon}$, where the function $w_{2}^{\varepsilon}$ is the unique solution, in $W^{1}\left(\Omega_{\varepsilon}^{\prime}\right)$, of the exterior Neumann problem

$$
\begin{cases}-\Delta w_{2}^{\varepsilon}=f & \text { in } \Omega_{\varepsilon}^{\prime}, \\ \frac{\partial w_{2}^{\varepsilon}}{\partial n_{\varepsilon}}=-\frac{\partial w_{1}}{\partial n_{\varepsilon}} & \text { on } \Gamma_{\varepsilon}, \\ w_{2}^{\varepsilon}(\boldsymbol{x})=O\left(|\boldsymbol{x}|^{-1}\right) & |\boldsymbol{x}| \rightarrow \infty .\end{cases}
$$

Finally, let $w_{2}$ denote the unique solution in $W^{1}\left(\mathbb{R}^{3}\right)$ of

$$
\begin{cases}-\Delta w_{2}=f & \text { in } \mathbb{R}^{3} \\ w_{2}(\boldsymbol{x})=O\left(|\boldsymbol{x}|^{-1}\right), & |\boldsymbol{x}| \rightarrow \infty\end{cases}
$$

As it is classical (see [8] for instance) the function $w_{2}$ is given by

$$
w_{2}(\boldsymbol{x})=\frac{1}{4 \pi} \int_{\mathbb{R}^{3}} \frac{f(\boldsymbol{y})}{|\boldsymbol{x}-\boldsymbol{y}|} d \boldsymbol{y}, \quad \boldsymbol{x} \in \mathbb{R}^{3} .
$$

Summarizing the decomposition process of the solution to Problem (5), we have

$$
u^{\varepsilon}=v+w_{1}+w_{2}^{\varepsilon} \quad \text { in } \Omega_{\varepsilon}^{\prime} \backslash \Sigma,
$$

where $v, w_{1}$ and $w_{2}^{\varepsilon}$ are solutions of (7), (9) and (10) respectively.

We now state our main result.

Theorem 3.1 Let $u^{\varepsilon}$ be the solution of Problem (5) and let $L^{\varepsilon}$ be the inductance coefficient defined by (6). Let $u$ be the function defined in $\mathbb{R}^{3} \backslash \Sigma$ by $u=v+w_{1}+w_{2}$, where $v, w_{1}$ and $w_{2}$ are solutions of (7), (9) and (11) 
respectively. Then, for every $\eta>0$,

$$
\begin{aligned}
\| u- & u^{\varepsilon} \|_{W^{1}\left(\Omega_{\varepsilon}^{\prime}\right)}=O\left(\varepsilon^{\frac{5}{6}-\eta}\right), \\
L^{\varepsilon}= & -\frac{\ell_{\gamma}}{2 \pi} \ln \varepsilon+L^{\prime}-\int_{\mathbb{R}^{3}} f\left(w_{1}+w_{2}\right) d \boldsymbol{x} \\
& +\int_{\Sigma}(1-\varphi)\left(\frac{\partial w_{1}}{\partial n}+\frac{\partial w_{2}}{\partial n}+2 \frac{\partial v}{\partial n}\right) d \sigma+O\left(\varepsilon^{\frac{5}{6}-\eta}\right),
\end{aligned}
$$

where $\ell_{\gamma}$ is the length of the curve $\gamma$ and

$$
L^{\prime}=\frac{\ell_{\gamma}}{2 \pi} \ln \frac{\delta}{2}+\frac{1}{4 \pi^{2}} \int_{\widehat{\Omega}}\left(a_{0} \xi \theta^{2}\left(\widehat{\varphi}^{\prime}\right)^{2}+\frac{\delta^{2} \xi \tau^{2}}{a_{0}} \widehat{\varphi}^{2}\right) d \widehat{\boldsymbol{x}}+\ell_{\gamma} \int_{\frac{1}{2}}^{1} \frac{\widehat{\varphi}^{2}}{2 \pi \xi} d \xi
$$

The next section is devoted to the proof of this result.

\subsection{Proof of error estimate}

Let $\widetilde{w}_{2}^{\varepsilon}=w_{2}^{\varepsilon}-w_{2}$. Clearly $\widetilde{w}_{2}^{\varepsilon}=u^{\varepsilon}-u, \widetilde{w}_{2}^{\varepsilon} \in W^{1}\left(\Omega_{\varepsilon}^{\prime}\right)$ and it satisfies

$$
\begin{cases}\Delta \widetilde{w}_{2}^{\varepsilon}=0 & \text { in } \Omega_{\varepsilon}^{\prime}, \\ \frac{\partial \widetilde{w}_{2}^{\varepsilon}}{\partial n_{\varepsilon}}=-\frac{\partial w_{1}}{\partial n_{\varepsilon}}-\frac{\partial w_{2}}{\partial n_{\varepsilon}} & \text { on } \Gamma_{\varepsilon}, \\ \widetilde{w}_{2}^{\varepsilon}(\boldsymbol{x})=O\left(|\boldsymbol{x}|^{-1}\right), & |\boldsymbol{x}| \rightarrow+\infty\end{cases}
$$

To estimate the solution of Problem (12), we need the following result.

Lemma 3.1 There is a constant $C$, independent of $\varepsilon$, such that :

$$
\begin{aligned}
& \|\psi\|_{L^{2}\left(\Gamma_{\varepsilon}\right)} \leq C \varepsilon^{\frac{1}{2}}|\ln \varepsilon|^{\frac{1}{2}}\|\psi\|_{W^{1}\left(\Omega_{\varepsilon}^{\prime}\right)} \quad \text { for all } \psi \in W^{1}\left(\Omega_{\varepsilon}^{\prime}\right) \\
& \|\psi\|_{L^{2}\left(\Gamma_{\varepsilon}\right)} \leq C\left(\varepsilon^{\frac{1}{2}}\|\psi\|_{W^{1, p}\left(\Omega_{\varepsilon}^{\prime}\right)}+\varepsilon^{\frac{4}{3}-\frac{2}{p}}\|\nabla \psi\|_{L^{p}\left(\Lambda_{\varepsilon}^{\delta}\right)}\right) \\
& \quad \text { for all } \psi \in W^{1, p}\left(\Omega_{\varepsilon}^{\prime}\right) \text { with compact support, } \frac{3}{2}<p<2 .
\end{aligned}
$$

For the proof we refer to [6]. 
Using the variational formulation associated with (12), Cauchy-Schwarz inequality and Estimate (13), we deduce

$$
\begin{aligned}
\int_{\Omega_{\varepsilon}^{\prime}}\left|\nabla \widetilde{w}_{2}^{\varepsilon}\right|^{2} d \boldsymbol{x} & =\int_{\Gamma_{\varepsilon}}\left(\frac{\partial w_{1}}{\partial n_{\varepsilon}}+\frac{\partial w_{2}}{\partial n_{\varepsilon}}\right) \widetilde{w}_{2}^{\varepsilon} d \sigma \\
& \leq\left\|\frac{\partial w_{1}}{\partial n_{\varepsilon}}+\frac{\partial w_{2}}{\partial n_{\varepsilon}}\right\|_{L^{2}\left(\Gamma_{\varepsilon}\right)}\left\|\widetilde{w}_{2}^{\varepsilon}\right\|_{L^{2}\left(\Gamma_{\varepsilon}\right)} \\
& \leq C \varepsilon^{\frac{1}{2}}|\ln \varepsilon|^{\frac{1}{2}}\left(\left\|\frac{\partial w_{1}}{\partial n_{\varepsilon}}\right\|_{L^{2}\left(\Gamma_{\varepsilon}\right)}+\left\|\frac{\partial w_{2}}{\partial n_{\varepsilon}}\right\|_{L^{2}\left(\Gamma_{\varepsilon}\right)}\right)\left\|\nabla \widetilde{w}_{2}^{\varepsilon}\right\|_{L^{2}\left(\Omega_{\varepsilon}^{\prime}\right)} .
\end{aligned}
$$

Using the integral representation of $w_{1}$, we easily check that

$$
\left\|\frac{\partial w_{1}}{\partial n_{\varepsilon}}\right\|_{L^{\infty}\left(\Gamma_{\varepsilon}\right)} \leq C
$$

Therefore

$$
\left\|\frac{\partial w_{1}}{\partial n_{\varepsilon}}\right\|_{L^{2}\left(\Gamma_{\varepsilon}\right)} \leq C\left(\operatorname{meas} \Gamma_{\varepsilon}\right)^{\frac{1}{2}} \leq C_{1} \varepsilon^{\frac{1}{2}} .
$$

To estimate $\frac{\partial w_{2}}{\partial n_{\varepsilon}}$, we use standard regularity results for elliptic problems, see [9], p. 343, to deduce, since $f \in L^{p}\left(\mathbb{R}^{3}\right)$ for $p<2$, that $w_{2} \in W_{\text {loc }}^{2, p}\left(\mathbb{R}^{3}\right)$. Then we apply Estimate (14) to the function $u=\frac{\partial w_{2}}{\partial x_{i}}, 1 \leq i \leq 3$ with $p=2-\eta$, $0<\eta<\frac{1}{2}$

$$
\left\|\frac{\partial w_{2}}{\partial x_{i}}\right\|_{L^{2}\left(\Gamma_{\varepsilon}\right)} \leq C\left(\varepsilon^{\frac{1}{2}}\left\|\frac{\partial w_{2}}{\partial x_{i}}\right\|_{W^{1, p}\left(\Omega_{\varepsilon}^{\prime}\right)}+\varepsilon^{\frac{1}{3}-\frac{\eta}{2-\eta}}\left\|\frac{\partial}{\partial x_{i}} \nabla w_{2}\right\|_{L^{p}\left(\Lambda_{\varepsilon}^{\delta}\right)}\right) .
$$

Since both norms on the right-hand side of the above inequality are uniformly bounded and since the outward unit normal $n_{\varepsilon}$ is uniformly bounded we obtain

$$
\left\|\frac{\partial w_{2}}{\partial n_{\varepsilon}}\right\|_{L^{2}\left(\Gamma_{\varepsilon}\right)} \leq C \varepsilon^{\frac{1}{3}-\frac{\eta}{2-\eta}} .
$$

Substituting (16) and (17) into (15) and using the inequality $|\ln \varepsilon| \leq C \varepsilon^{-2 \eta}$, we get

$$
\int_{\Omega_{\varepsilon}^{\prime}}\left|\nabla \widetilde{w}_{2}^{\varepsilon}\right|^{2} d \boldsymbol{x} \leq C_{1} \varepsilon^{\frac{5}{6}-\frac{\eta}{2-\eta}-\eta}\left\|\nabla \widetilde{w}_{2}^{\varepsilon}\right\|_{\boldsymbol{L}^{2}\left(\Omega_{\varepsilon}^{\prime}\right)}
$$

Therefore

$$
\left\|\nabla \widetilde{w}_{2}^{\varepsilon}\right\|_{L^{2}\left(\Omega_{\varepsilon}^{\prime}\right)} \leq C_{2} \varepsilon^{\frac{5}{6}-\eta} \quad \text { for all } \eta>0
$$




\subsection{Proof of asymptotic expansion}

Using the decomposition $u^{\varepsilon}=v+w^{\varepsilon}=v+w_{1}+w_{2}^{\varepsilon}$ it follows

$$
L^{\varepsilon}=\int_{\Omega_{\varepsilon}^{\prime} \backslash \Sigma}|\nabla v|^{2} d \boldsymbol{x}+\int_{\Omega_{\varepsilon}^{\prime} \backslash \Sigma}\left|\nabla w^{\varepsilon}\right|^{2} d \boldsymbol{x}+2 \int_{\Omega_{\varepsilon}^{\prime} \backslash \Sigma} \nabla v \cdot \nabla w^{\varepsilon} d \boldsymbol{x} .
$$

Using Green's formulae, we can write $L^{\varepsilon}$ in the form

$$
L^{\varepsilon}=\int_{\Omega_{\varepsilon}^{\prime} \backslash \Sigma}|\nabla v|^{2} d \boldsymbol{x}-\int_{\Omega_{\varepsilon}^{\prime}} f w^{\varepsilon} d \boldsymbol{x}+\int_{\Sigma}(1-\varphi)\left(\frac{\partial w^{\varepsilon}}{\partial n}+2 \frac{\partial v}{\partial n}\right) d \sigma .
$$

Using the previous estimate for $w_{2}^{\varepsilon}$ and some regularity results $\left(w_{2} \in W^{2, p}\left(\Omega_{\delta}\right)\right.$, $w_{1} \in H^{2}\left(\Omega_{\frac{\delta}{2}}\right)$ ), we can estimate each term in (18), to obtain for all $\eta>0$,

$$
L^{\varepsilon}=\int_{\Omega_{\varepsilon}^{\prime} \backslash \Sigma}|\nabla v|^{2} d \boldsymbol{x}-\int_{\mathbb{R}^{3}} f w d \boldsymbol{x}+\int_{\Sigma}(1-\varphi)\left(\frac{\partial w}{\partial n}+2 \frac{\partial v}{\partial n}\right) d \sigma+O\left(\varepsilon^{\frac{5}{6}-\eta}\right),
$$

where $w=w_{1}+w_{2}$.

To complete the result, an explicit calculation yields

$$
\int_{\Omega_{\varepsilon}^{\prime} \backslash \Sigma}|\nabla v|^{2} d \boldsymbol{x}=-\frac{\ell_{\gamma}}{2 \pi} \ln \varepsilon+L^{\prime}+O(\varepsilon),
$$

where $\ell_{\gamma}$ is the length of the curve $\gamma$ and

$$
L^{\prime}=\frac{\ell_{\gamma}}{2 \pi} \ln \frac{\delta}{2}+\frac{1}{4 \pi^{2}} \int_{\widehat{\Omega}}\left(a_{0} \xi \theta^{2}\left(\widehat{\varphi}^{\prime}\right)^{2}+\frac{\delta^{2} \xi \tau^{2}}{a_{0}} \widehat{\varphi}^{2}\right) d \widehat{\boldsymbol{x}}+\frac{\ell_{\gamma}}{2 \pi} \int_{\frac{1}{2}}^{1} \frac{\widehat{\varphi}^{2}}{\xi} d \xi .
$$

\section{References}

[1] R. Touzani, Analysis of an eddy current problem involving a thin inductor, Comp. Methods Appl. Mech. Eng., Vol. 131 (1996) 233-240.

[2] A. Bossavit, Electromagnétisme en vue de la modélisation, Springer-Verlag (1988).

[3] A. Bossavit, J.C. Vérité, The TRIFOU Code : Solving the 3-D eddy-current problem by using $h$ as a state variable, IEEE Transactions on Magnetics, MAG19, No. 6, (1983) 2465-2470.

[4] R. Dautray, J.L. Lions, Analyse mathématique et calcul numérique pour les sciences et les techniques, Tome 2, Masson, Paris (1985).

[5] L. Landau, E. Lifshitz, Electrodynamics of Continuous Media, Pergamon, London (1960). 
[6] Y. Amirat, R. Touzani, Asymptotic behaviour of the inductance coefficient for thin conductors, Preprint.

[7] E. Kreyszig, Differential Geometry, Dover Publications, New York (1991).

[8] J.C. Nedelec, Approximation des équations intégrales en mécanique et en physique, Centre de Mathématiques Appliquées, Ecole Polytechnique Palaiseau (1977).

[9] R. Dautray, J.L. Lions, Analyse mathématique et calcul numérique pour les sciences et les techniques, Tome 1, Masson, Paris (1984). 